\title{
Methodology for Sizing the Solar Field for Parabolic Trough Technology with Thermal Storage and Hybridization
}

\author{
Suresh N S ${ }^{\mathrm{a}}$, Thirumalai N C ${ }^{\mathrm{b}}$, Badri S Rao ${ }^{\mathrm{c}}$, M.A. Ramaswamy ${ }^{\mathrm{d}}$
}

${ }^{a}$ Corresponding Author:

Research Engineer,

Phone: +91(80) 66902536, Mobile: +91-9663819468, E-mail: suresh@cstep.in

${ }^{\mathrm{b}}$ Research Scientist,

Phone: +91(80) 66902505, Mobile: +91-9740744066, E-mail: thirumalai@cstep.in

${ }^{\mathrm{c}}$ Research Engineer,

Phone: +91(80) 66902537, Mobile: +91-9986618433, E-mail: badri@cstep.in

${ }^{\mathrm{d}}$ Advisor,

Phone: +91(80) 66902554, Mobile: +91-9845411292, E-mail: mar@cstep.in

Address:

Center for Study of Science Technology and Policy, $10^{\text {th }}$ Cross, Papanna Layout, Mayura Street, Nagashettyhalli, RMV II Stage,

Bangalore-560 094, Karnataka, INDIA

\begin{abstract}
A detailed methodology to design the size of solar field for a parabolic trough plant is not explicitly available in open literature, particularly if thermal storage and hybridization are also considered, as most of the papers present a gross overview. This paper gives a procedure to determine the annual electricity generated for a parabolic trough based solar plant of a given rated capacity (1-50 MWe), at a chosen location \& given hourly annual solar input, specified hours of thermal energy storage using a two-tank molten salt system and specified fraction of hybridization using natural gas. In this methodology losses due to shut down or cloud cover are also covered. The size of the solar field is optimized for the maximum annual solar to electric conversion efficiency using the concept of solar multiple (ratio of actual aperture area to the reference aperture area needed to get rated power output at maximum solar input). This procedure is validated with the existing parabolic trough plants (Solar Energy Generating Systems VI and Solana Generating Station) and it was found that the annual electrical energy generated by the plant matches reasonably well.

Jodhpur, in India, was considered as a location for the case study and the results are presented to understand the influence of thermal storage and hybridization for a given capacity of the plant. The results for various combinations of thermal storage hours and fraction of hybridization used with respect to plant capacity, solar multiple, annual plant efficiency etc. have been discussed in detail. It is observed from the results that, under design conditions, the reference aperture area per MW decreases as plant capacity increases and reaches a limiting value asymptotically at a capacity of $50 \mathrm{MW}$. The optimized size of the solar field, with respect to annual efficiency, is found to be 1.4 and 2.3 times the size under design conditions for zero and six hours thermal storage respectively. The benefit of hybridization is high for lower solar multiples.
\end{abstract}

Keywords: Parabolic Trough; Thermal Storage; Hybridization; Concentrated Solar Power; Solar Multiple;

$\begin{array}{ll}\text { Nomenclature } & \text { Actual mirror aperture area } \\ A_{a} & \text { Reference mirror aperture area } \\ A_{r} & \text { Chord of the mirror } \\ \mathrm{C} & \text { Thermal energy available from storage } \\ E_{t e a} & \text { Maximum amount of thermal energy that can be stored } \\ E_{t e s, \max } & \text { Loss factor } \\ L_{f} & \text { Actual gross power generated } \\ P_{g} & \text { Rated gross power } \\ P_{g, d} & \end{array}$

(C) 2014. This manuscript version is made available under the Elsevier user license http://www.elsevier.com/open-access/userlicense/1.0/ 
$P_{a b s}$

$P_{a b s, d}$

$P_{h b}$

$P_{\text {htf }}$

$P_{h t f, d}$

$P_{h t f, s}$

$P_{t h, d}$

$P_{t h, s, d}$

SM

$e_{g}$

$e_{g, a}$

$e_{g, t}$

$e_{\text {grid }}$

$e_{\text {grid, } t}$

$e_{h b}$

$e_{h b, t}$

$e_{s}$

$e_{s, t}$

$e_{\text {start }}$

$f_{h b}$

$f_{\text {hb,used }}$

$f_{\text {th }}$

$f_{p}$

$f_{p a}$

$f_{t h, s}$

$f_{t h, s t}$

$f_{t h, s t a}$

$t_{s}$

$\beta$

$\gamma$

$\delta$

$\eta_{a b s}$

$\eta_{a b s, d}$

$\eta_{c}$

$\eta_{h e}$
Thermal power impinging on the absorber tube

Thermal power impinging on the absorber tube at design conditions

Maximum thermal power input to HTF from hybridization

Thermal power input from HTF to heat exchanger

Thermal power input from HTF to heat exchanger at design conditions

Thermal power input from solar field to HTF

Thermal power of working fluid at design conditions

Solar power input to mirrors at design conditions

Solar Multiple

Gross electrical energy generated without considering energy needed for start-up

Gross electrical energy generated accounting for start-up

$\sum e_{g, a}$

Electrical energy supplied to grid

$\sum e_{\text {grid }}$

Electrical energy apportioned to hybridization

$\sum e_{h b}$

Electrical energy apportioned to solar input

$\sum e_{S}$

Equivalent electrical energy required for start-up accounting for thermal losses during shut down period

Maximum fraction of hybridization power permitted

Actual hybridization fraction used

Fraction of thermal power delivered to power block

Fraction of the gross electrical power generated ignoring thermal losses during shutdown

Fraction of gross electrical power generated taking into account thermal losses during shutdown

Solar thermal power as a fraction of the design thermal power

Fraction of thermal power used from storage

Fraction of thermal power available from storage

Number of hours of thermal storage

Angle of tilt of parabolic trough

Intercept factor

Declination of the day

Absorber efficiency

Efficiency of absorber tube at design conditions

Efficiency of solar collection

Efficiency of power block heat exchanger 
$\eta_{m}$

$\eta_{p, d}$

$\eta_{p l}$

$\eta_{r}$

$\eta_{s-e}$

$\eta_{s t}$

$\theta$

$\rho$

$\phi$

$\omega$

Optical efficiency of the mirror system

Power block efficiency at design conditions

Part load efficiency of power block

Relative efficiency $=\eta_{p l} / \eta_{p, d}$

Annual efficiency attributed to the solar

Efficiency of storage heat exchanger

Angle between the normal to the mirror aperture and sun's rays

Specular reflectivity of the mirror

Latitude of the location

Solar hour angle 


\section{Introduction}

Among the Concentrated Solar Power (CSP) technologies, Parabolic Trough (PT) technology is the most commercially proven technology (Hachicha et al, 2013; Abdelkader Zaaraoui 2012; Reddy, 2012). A PT power plant can be segregated into two major segments: solar field and power block. The schematic representation of the parabolic trough plant with thermal storage and hybridization is shown in Fig 1. In Fig. 1, T-1 to T-4 represents stages of turbines and other components are self explanatory in the figure itself. The solar field consists of highly reflective mirrors mounted on a support structure, which can be tilted about an axis (normally aligned in the North-South direction) to track the sun as it moves from East to West. At the focal line of the parabolic mirrors, a receiver is mounted. Receiver composed of absorber tube and glass envelope. Absorber tube in the receiver is encapsulated in a glass envelope and the annular space between the glass cover inner surface and the absorber is evacuated. The absorber tube is given a special coating which along with the evacuation leads to better absorption and transfer of heat to the Heat Transfer Fluid (HTF) flowing inside the receiver (Ravi, 2009; Thomas 1992). The HTF flows in and out from the absorber tubes through header pipes. The power block is almost similar to that used in the conventional power plants. In the power block, the thermal energy acquired by the HTF is used to operate a conventional steam turbine to generate electricity. Generally, the thermal energy of the HTF is transferred to the feed water through a series of heat exchangers (pre-heating, vaporizing and super-heating) to produce superheated steam, which drives the steam turbine coupled to a generator (Rovira et al, 2013; Montes et al, 2009). The steam exiting the turbine is condensed using wet or dry cooling condenser and the condensed water goes to the feed water pumps to pump it to the heat exchanger.

Thermal storage is necessary to provide full-load, steady state electrical generation during time of cloud cover, beyond sunset for durations on the scale of hours, and to assure predictable delivery to meet peak demand (Kuravi 2013; Montes et al, 2011; Powell, 2012; Ravaghi-Ardebili, 2013). The maximum amount of thermal storage is dependent on the capacity of the hot and cold tanks and the thermal properties of the storage medium. Hybridization, as defined for this study, refers to the use of other fuels such as natural gas, coal, lignite and biomass, to supplement the solar radiation. Typically, hybridization in a CSP plant is used for taking care of transients and hence requires a quick response. Natural gas is a preferred fuel for this purpose (Pilkington, 1996; Gharbi, 2011).

Numerous studies have been carried out to evaluate the performance of parabolic trough technology for power generation (Feldhoff et al, 2012; Fichtner, 2010; Herrmann et al, 2004; Lippke F, 1995; Montes et al, 2009; Patnode A.M, 2006). Herrmann et al, (2004) deals with the merits of molten salt vs. HTF itself as a storage medium in parabolic trough thermal plant based on the experiences of SEGS and Solar Two plants. Montes et al, (2009) discusses the optimization of solar field based on LCOE for a stand-alone solar parabolic trough power plant using oil as HTF without considering hybridization and thermal storage. Garcia et al (2011) presents a detailed model to estimate the performance of parabolic trough power plant with thermal storage. This paper facilitates the prediction of the electricity output by considering the detailed component level analysis in the solar field. Feldhoff et al, (2012) carried out a comparison of two reference parabolic trough plants (one with direct steam generation and other with synthetic oil) of capacity $100 \mathrm{MWe}$ with integrated thermal storage especially in terms of economic potential. The study tells that the LCOE by direct steam generation mode is higher compared to that of synthetic oil based plant integrated with storage.

All the previous works excluding Montes et al, (2009) referred to deal mostly with the performance aspects of specific plants, some of them in much more detailed than what has been considered in the present paper but none of them deal with how to arrive at an optimum solar field size, even for a single parameter. Even in Montes et al (2009), the paper neither gives the details about the efficiency of various components in the plant nor about the procedure used for estimating the annual electrical energy generated and it is specific to only $50 \mathrm{MWe}$ plant capacity.

Ours is the first paper, using hourly simulation model, to arrive at the optimized solar field based on the annual solar to electric efficiency considering the combination of factors such as solar multiple, thermal storage, hybridization by considering all the components efficiencies in the solar field and the power block. Also, we considered the estimation of reference aperture area of the solar field taking into account the $(D N I \times \cos \theta)_{\max }$ available over a year at the chosen location. Here $D N I$ represents Direct Normal Irradiance and $\theta$ represents the angle of incidence. The present model has a primary objective to estimate the annual electrical energy generated for a given solar field, based on typical efficiencies for various elements of the plant and further arrive 
at the optimum solar field size based on annual solar to electric conversion efficiency. Since the emphasis in the present paper is on the design, the design has to be based on Typical Meteorological Year (TMY) solar data, which is some sort of an average covering for many years, rather than data of any particular year. Of course, due to vagaries of nature, the performance will vary from year to year, which cannot be avoided. The results from our methodology, gives a better understanding of the effect of various design and operating parameters to assess the solar field size and performance of parabolic trough power plant. The methodology is also validated with existing parabolic trough plants such as SEGS-VI and Solana station.

\section{Methodology for technical assessment of parabolic trough CSP plant}

A methodology for an hourly simulation model has been developed. This methodology considers the performance of the plant both under design and off-design conditions.

\subsection{Overview of design parameters}

The overview of design parameters such as reference aperture area and solar multiple along with the thermal storage, hybridization and loss factors are explained below. All these are included in developing the model to estimate the annual electricity generation.

\subsubsection{Estimation of reference aperture area}

In the first step, for a given location, the mirror aperture area of the solar field required to generate the rated gross capacity of the power block is estimated. This is done for the condition of maximum solar power, $(D N I \times \cos \theta)_{\max }$ incident on the mirrors. This mirror aperture area is called the Reference Mirror Aperture Area $\left(A_{R}\right)$. The reference solar field is based purely on the maximum solar power available and is independent of thermal storage or hybridization. It is obvious, that if this reference solar field is used, then the rated power is generated only for a brief period corresponding to $(D N I \times \cos \theta)_{\max }$. At other times, the power generated is below to the rated capacity. Consequently, the annual electrical energy generated will not make the plant economically viable. The design essentially boils down, to determine the factor called solar multiple by which the reference area has to be increased for getting the least $L C O E$. So, we need to first determine the reference aperture area.

The step by step procedure for estimation of reference mirror aperture area $\left(A_{R}\right)$ is given below:

a. For the chosen location, determine $(D N I \times \cos \theta)_{\max }$ from the hourly $D N I$ data of the year

b. Consider a gross capacity of power block, $P_{g, d}$

c. Estimate the thermal power input to the working fluid $\left(P_{t h, d}\right)$ taking into account the efficiency of the power block at design conditions $\left(\eta_{p, d}\right)$

d. Calculate the thermal power input to be given to the HTF $\left(P_{h t f, d}\right)$ considering the efficiency of the heat exchanger $\left(\eta_{h e}\right)$.

e. Determine the efficiency of the receiver $\left(\eta_{a b s, d}\right)$ corresponding to the solar input to the receiver per unit length at $(D N I \times \cos \theta)_{\max }$

f. Calculate solar power $\left(P_{a b s, d}\right)$ required to be incident on the receiver.

g. Determine the reference mirror aperture area $\left(A_{R}\right)$ using $(D N I \times \cos \theta)_{\max }$ and the optical efficiency of the mirror $\left(\eta_{\text {mirror }}\right)$.

\subsubsection{Solar Multiple}

Solar Multiple $(S M)$ is defined as the ratio of the actual aperture area $\left(A_{a}\right)$ of the mirrors to the reference mirror aperture area $\left(A_{R}\right)$ (Montes et al, 2009). It generates the rated electrical power corresponding to $(D N I \times \cos \theta)_{\max }$ for the chosen location. In the present paper, the SM is optimised for maximum annual solar to electric conversion efficiency $\left(\eta_{s-e}\right)$. If $\left(A_{a}\right)$ is equal to $\left(A_{R}\right), S M=1.0$, then the design power is generated only over a very short period. At all other times, solar thermal input to the plant is lower than the design value and the power block would be working under part load. The efficiency of the plant under part load will be low. Thus the combined effect of lower solar thermal input and lower efficiency during most of the time results in a relatively low annual electrical energy generated. On the other hand, if one chooses to have a solar field with higher than the optimum value of SM, then the plant will be operating most of the time at full load. However, if thermal 
storage is not provided, the plant will not be able to utilize the excess solar power and some mirrors will have to be defocused. In the present model, various values of SM are considered and the corresponding annual electrical energy is computed for chosen values of storage and hybridization considering loss factor.

\subsubsection{Thermal Storage}

A major advantage of CSP over Photovoltaic is the possibility of providing thermal storage (Hermann 2012; IRENA, 2012). This is to provide power during the evening and night and also to tide over intermittent periods of cloud cover. As stated above, when $S M>1$ and in absence of thermal storage, when $(D N I \times \cos \theta)$ is close to the design value, some mirrors have to be defocused and consequently the solar thermal energy, which could have been utilized, is wasted. Thus in CSP plants, it is better to provide a few hours of thermal storage $\left(t_{s}\right)$. Therefore, it can deliver $t_{s}$ hours of the rated electrical power, even when there is no thermal input from the sun.

\subsubsection{Hybridization}

Hybridization in a CSP plant is generally used for taking care of transients, and hence requires a quick response. Thus, natural gas is a preferred fuel for hybridization (Pilkington, 1996; Gharbi, 2011). Here again, the maximum amount of hybridization permitted is chosen as a fraction of the design thermal power that needs to be given to the HTF to generate the rated gross power.

In other words, if fraction of hybridization $\left(f_{h b}\right)$ is the hybridization permitted and if $P_{h t, d}$ is the thermal power of HTF needed to generate the design gross power, then the natural gas burner is designed to deliver to the HTF a maximum thermal power of $f_{h b} \times P_{h f, d}$.

\subsubsection{Loss Factor}

When a CSP plant is not operating during night and times of cloud cover, the temperature of HTF in the absorber tubes will fall below the operating levels due to thermal loss. Hence, before the plant can be restarted and brought into operation, the solar energy would first be utilized to make up the thermal losses and bring back the HTF to operating conditions. Obviously, longer the period of shutdown, greater will be the thermal loss. If the insulation were perfect and there were no thermal losses, this solar energy which is now used for making up the thermal loss, would have produced electrical energy.

Thermal losses depend on ambient conditions, such as ambient temperature, wind velocity and various other design parameters of the plant. When the plant is started after a period of shut down, the thermal energy which the HTF would have lost during shutdown would have to be made up before it can be passed on to the heat exchanger of the power block to generate electrical energy. Thus there is not only a lag in electrical energy generation relative to the solar energy input but also the loss in the electrical energy generated relative to what it would have generated in the absence of thermal loss. Therefore for the assessment of CSP plant at preliminary stage we can directly consider this thermal energy loss in terms of electrical energy loss for every hour of nonoperation of the plant. So we define a loss factor $L_{f}$, as the fraction of the electrical energy lost (due to thermal losses) per hour of shutdown, to the energy that the plant would have generated in one hour at rated capacity. If the plant of rated power $P_{g, d}$, is shut down for $t$ hours then the electrical energy lost will be $L_{f} \times t \times P_{g, d}$.

\subsection{Inputs for technical assessment of parabolic trough based CSP system}

The inputs required for the technical assessment are as follows:

\subsubsection{Hourly DNI data for a Typical Meteorological Year (TMY) for the chosen location}

For a reliable assessment of CSP plant, DNI data at close intervals of time throughout a Representative Year (RY) or $T M Y$ from measured ground data over several years are required. However, for a preliminary assessment of CSP plant, at least an hourly $D N I$ data for a $T M Y$ is required.

\subsubsection{Efficiency of solar collection}


The solar collection efficiency $\eta_{c}$ is defined as the ratio of actual solar power incident on the mirrors to the maximum possible power that could be captured (normal incidence).

Actual solar power, $P_{\text {actual }}$ is given by

$$
P_{\text {actual }}=\sum_{i=1}^{N} D N I \times A_{i} \times \cos \theta_{i}
$$

where, $\mathrm{N}$ is the number of mirrors, $A_{i}$ is the aperture area of the $i^{\text {th }}$ mirror element and $\theta_{i}$ is the angle between the normal to the $i^{\text {th }}$ mirror element and sun's rays. Therefore

$$
\eta_{c}=\frac{\sum_{i=1}^{N} D N I \times A_{i} \times \cos \theta_{i}}{\sum_{i=1}^{N} A_{i}}
$$

For a parabolic trough system aligned in the North-South direction, which is the most common, $\theta_{i}$ is given by

$$
\cos \theta_{i}=\sin \phi \sin \delta \cos \beta+\cos \phi \cos \delta \cos \omega \cos \beta+\cos \delta \sin \omega \sin \beta
$$

where,

$\phi$ is the latitude of the location

$\delta$ is the declination, which depends on the day of the year

$\omega$ is the hour angle

$\beta$ is the angle of tilt of parabolic trough

The angle of the tilt $(\beta)$ can be found by

$$
\tan \beta=\frac{\cos \delta \sin \omega}{\sin \phi \sin \delta+\cos \phi \cos \delta \cos \omega}
$$

Since all the troughs are tilted by the same amount of $\beta$ and $\theta_{i}$ is same for all troughs. So $\eta_{c}=\cos \theta_{i}$ and can be taken for the whole aperture area.

\subsubsection{Efficiency of mirror system}

The optical efficiency of the mirror system, $\eta_{m}$ may be defined as follows

$$
\eta_{\mathrm{m}}=\frac{\text { Solar power incident on the absrober }}{\text { Solar power incident on the mirror }}
$$

$\eta_{\mathrm{m}}$ depends on various factors as indicated below (Forristall, 2003):

where,

$$
\eta_{m}=\varepsilon_{1}^{\prime} \varepsilon_{2}^{\prime} \varepsilon_{3}^{\prime} \varepsilon_{4}^{\prime} \varepsilon_{5}^{\prime} \varepsilon_{6}^{\prime} \rho_{c l} K
$$

$\varepsilon_{1}^{\prime}=$ HCE Shadowing

$\varepsilon_{2}^{\prime}=$ Tracking error (bellows, shielding, supports)

$\varepsilon_{3}^{\prime}=$ Geometry error (mirror alignment)

$\rho_{c l}=$ Clean mirror reflectance

$\varepsilon_{4}^{\prime}=$ Dirt on mirrors $=$ Reflectivity $/ \rho_{c l}$

$\varepsilon_{5}^{\prime}=$ Dirt on $\mathrm{HCE}$

$\varepsilon_{6}^{\prime}=$ Unaccounted error

$\mathrm{K}=$ Incident angle modifier, $\cos \theta+0.000884 \theta-0.00005369 \theta^{2}$

In this paper representative values (Forristall, 2003) are used but in applying this methodology for any specific design, the corresponding values have to be used.

With the solar field aperture area and other parameters given in 2.2.1 and 2.2.2, the thermal power impinging on the absorber tube can be calculated. This is given by

$$
P_{a b s}=\eta_{c} \times \eta_{m} \times D N I \times A_{a}
$$




\subsubsection{Efficiency of receiver}

Receiver efficiency ( $\eta_{\text {receiver }}$ ) is the ratio of the thermal power transferred to the HTF to the thermal power incident on the receiver. Most of the CSP plants with parabolic trough system use the SCHOTT's selectively coated $70 \mathrm{~mm}$ stainless steel tube enclosed in $115 \mathrm{~mm}$ evacuated glass tube, each segment being $4 \mathrm{~m}$ long (Forristall, 2003). This is assumed as the benchmark for evaluation of a CSP plant using trough technology. The thermal properties of the absorber tube and the glass tube are known and they can be used to evaluate the $\eta_{\text {receiver }}$ for a trough system (Forristall, 2003; Suresh et al, 2014). Also most plants use Therminol VP-1 as HTF (Franchini G, 2013). The inlet and outlet temperatures of the HTF using synthetic oil and steam Rankine cycle get fixed from considerations of the flash point of the synthetic oil and the saturation temperature of steam. Therefore in most plants, the maximum outlet temperature of $\mathrm{HTF}$ is limited to $390^{\circ} \mathrm{C}$ while the inlet temperature is taken to be $290^{\circ} \mathrm{C}$ (Pilkington, 1996; Giostri 2012).

The system is so designed that for the design conditions $(D N I \times \cos \theta)_{\max }$ and design mass flow, the HTF enters the receiver at $290^{\circ} \mathrm{C}$ and exits at $390^{\circ} \mathrm{C}$. Hence we need to estimate the $\eta_{\text {receiver,d }}$ under design conditions of thermal input to the receiver. However, if due to reduced $D N I$, the thermal input to the receiver gets reduced below the design value, then the normal practice is to adjust the mass flow of HTF so as to maintain the same inlet and outlet temperatures of HTF. Some variations in HTF outlet temperature may be permitted in actual operation. Under the off-design conditions of lower solar input, $\eta_{\text {receiver }}$ will be less than $\eta_{\text {receiver }, d}$ and we need to estimate the variation of $\eta_{\text {receiver }}$ with thermal input to the receiver. The details of the heat transfer analysis for a receiver of SCHOTT type are given in the report (Suresh et al, 2014).

The thermal loss from the absorber tube depends on the ambient conditions, viz., ambient temperature and wind velocity. $2 \%$ reduction in the efficiency is taken to account for end losses of the receiver in the loop. The efficiencies obtained from the steady state analysis were decreased (by $5 \%$ ) to account for these losses. Based on all these considerations, efficiency of the absorber tube as a function of the thermal power input per unit length $(\mathrm{kW} / \mathrm{m})$ was calculated and is shown in Fig.2 (Suresh et al, 2014; Forristall, 2003).

For a chosen chord width, $C(\mathrm{~m})$ of the trough and for a given location of the plant, the solar power input per meter to the absorber tube $(P s)$ is given by

$$
P_{s}(W / m)=C \times D N I \times \cos \theta_{i} \times \eta_{m}
$$

Corresponding to this thermal input $\eta_{\text {receiver }}$ is found out from Fig.2. Therefore the thermal power given to HTF is given by

$$
P_{h t f}=P_{s} \times \eta_{\text {receiver }}
$$

\subsubsection{Efficiency of heat exchanger}

There are two heat exchangers needed in a typical parabolic trough plant with thermal storage. One is between the HTF and the power block and other is between the HTF and thermal storage medium. The efficiency of both the heat exchangers is taken as $95 \%$ in the analysis (Montes et al, 2009).

\subsubsection{Efficiency of power block}

The efficiency of the power block ( $\eta_{p, d}$ ) depends on many factors such as the inlet pressure and temperature of steam, condenser pressure, mass flow rate of steam, capacity of the power block etc. We have confined the analysis to power blocks using steam as the working fluid. For the power blocks used in CSP plants, using synthetic oil as HTF, the variation of $\eta_{p, d}$ with capacity is shown in Fig.3. This applies for plants with wet cooling option. This is based on discussions, data from manufacturers and literature (Lovegrove et al, 2012; Montes et al 2009). It shows that the power block efficiency is very low at low capacities. It increases with the 
increase in capacity and plateaus at about $37 \%$ for a power block capacity of about $50 \mathrm{MW}$. This explains why steam cycle based CSP plants make economic sense at capacities of 50-100 MW.

Unlike conventional power plants, CSP plants also have to work under part load conditions. Hence, we need to know the variation in efficiency, $\eta_{p l}$ under part load conditions. We defined a relative efficiency

$\eta_{r}=\eta_{p l} / \eta_{p, d}$ and consider the variation of $\eta_{r}$ as function of gross fractional power generated, $f_{p}$, defined as a fraction of the full load capacity. Again based on discussions with manufacturers and available literature [Montes et al, 2009], $\eta_{r}$ vs. $f_{p}$ can be approximated as shown in Fig.4 (Lovegrove et al, 2012; Montes et al 2009).

The variation of $\eta_{r}$ vs. $f_{p}$ given in Fig.4 can be analytically represented as

$$
\eta_{r}=\frac{1.1 \times f_{p}}{f_{p}+0.1}
$$

To find $f_{p}$ under part load conditions, we need the relation between $f_{p} \& f_{t h}$.

$$
f_{t h}=\frac{P_{h t f}}{P_{h t f, d}}=\frac{\mathrm{P}_{\mathrm{g}, \mathrm{d}} \times f_{p} / \eta_{p, d} \times \eta_{r} \times \eta_{h e}}{P_{g, d} / \eta_{p, d} \times \eta_{h e}}
$$

Substituting for $\eta_{r}$ from Eq. (10) and simplifying, we get

$$
\begin{array}{ll}
f_{p}=1.1 f_{\text {th }}-0.1 & \text { for } 0.09 \leq f_{\text {th }} \leq 1.0 \\
f_{p}=f_{\text {th }} & \text { for } 1.0<f_{\text {th }} \leq 1.1
\end{array}
$$

The above relationship is shown in Fig.5.

Since power block efficiency reduces with part load, the fractional electrical power generated is less than the fractional thermal power input as is evident from Fig.5

\subsubsection{Thermal losses associated during plant shut down period}

An overview of how thermal losses are taken into account is given in section 2.1.5. The thermal losses that occur overnight are roughly equivalent to the energy that the plant would have generated over $3 / 4^{\text {ths }}$ of an hour. This is equivalent to the energy required to reheat the HTF (Gee and Murphy, 1981). Assuming that the plant was not operational for 15 hours overnight, we can roughly say that for every one hour of non-operation, the electrical energy, which would have to be used to make up the losses, is roughly equivalent to the energy that the plant would have produced for $1 / 20$ hour. In the above case, the loss factor is 0.05 and the same is considered in our analysis.

\subsubsection{Thermal storage}

In this study we have considered a two tank molten salt storage technology.

a. First choose the number of hours $\left(t_{s}\right)$ of thermal storage.

b. The efficiency of heat transfer from HTF to the molten salt is taken as $\eta_{s t}$ during charge and discharge. The default value is taken as 0.97 (Montes et al, 2009).

c. Therefore, if we need $t_{s}$ hours of thermal storage, the maximum amount thermal storage energy that need to be collected by the storage system is

$$
E_{\text {tes, } \max }=\frac{P_{h t f, d \times} t_{s}}{\eta_{s t}}
$$

\subsubsection{Hybridization}

It is assumed that hybridization is used to augment the thermal energy in the solar field whenever possible to maximize the electrical energy generated. The hierarchy of usage of thermal power is in this order: first is solar field, next is thermal storage and last is hybridization. If $P_{h t f, d}$ is the thermal power required to be given to HTF to generate the rated gross power and if $P_{h b}$ is the maximum thermal power that the natural gas boiler can 
augment through hybridization (Fig.1), then the extent of hybridization is defined by $f_{h b}$, which is the ratio of $P_{h b}$ to $P_{h f, d}$. In other words, $f_{h b}$ is the maximum fraction of thermal power that can be given to HTF through hybridization to the thermal power needed to generate the rated gross power.

\subsection{Engineering model for computation of Annual Electrical Energy Generated, Capacity Factor and Annual Plant Efficiency}

The engineering model for computing the annual electrical energy for a chosen SM, thermal storage hours and fraction of hybridization is explained in this section. It is a usual practice to permit operation of the power block at $10 \%$ more than the design capacity. Likewise, if the thermal power available falls below $25 \%$ of the design value, the plant is shut down.

Starting from the $1^{\text {st }}$ hour to the last hour of the year (8760), the following computations are carried out:

a) For the chosen SM, the actual aperture area of the mirrors $A_{a}$ is determined by multiplying the SM with reference aperture area.

b) The solar power impinging per unit length of the receiver $\left(P_{s}\right)$ is computed using Eq. (6).

c) Corresponding to this value of $P_{s}$, the thermal efficiency of the receiver $\eta_{\text {receiver }}$ is found out as explained in Section 2.2.4. Using this information, the solar thermal power transferred to HTF $P_{h t f, s}$ is computed.

d) Fractional thermal power from solar field, $f_{t h, s}$, the fraction of $P_{h t f, s}$ to $P_{h t, d}$ is computed.

e) $f_{\text {th }}$ is defined as the ratio of the thermal power delivered to the power block heat exchanger $\left(P_{h t}=D N I \times \cos \theta \times \eta_{m} \times A_{r} \times S M \times \eta_{\text {receiver }}\right)$ to the thermal power needed to generate rated gross power $\left(P_{h t, d}\right)$. When $f_{t h}=1.0$, the rated gross power is generated. Since power block is allowed to operate at $10 \%$ more than the design capacity, the maximum value of $f_{t h}$ is 1.1. $f_{t h}$

f) Depending on the value of $f_{t h, s}\left(=P_{h t, s, s}\left(P_{h t f, d}\right)\right.$ different options are chosen as given below.

i) If $f_{t h, s}$ is greater than 1.1, then HTF thermal energy equivalent to $f_{t h}=1.1$, (i.e., $P_{h t f}=1.1 \times P_{h t f, d}$ ) is sent to the heat exchanger to produce electrical power. The balance thermal energy is sent to the thermal storage system.

ii) If $f_{t h, s}$ is less than 1.1 and if stored thermal energy is available, it is used to augment $f_{t h}$ up to a value 1.1 for electricity generation.

iii) If $f_{t h}$ after augmenting with available stored energy $f_{t h, s t a}$, is less than 1.1, then it is further augmented with hybrid power (limited to the maximum permissible value ${ }^{1}$ ). Even if $f_{t h, s}$ is zero, the plant can be run with stored thermal energy and hybridization.

iv) If augmented value of $f_{t h}$, including stored thermal energy and hybridization is less than 0.25 , the plant is shutdown.

v) When the plant is shutdown, thermal losses would occur, which would have to be made up, before the plant can generate power. For $t$ hours of non-operation, electrical energy equivalent of thermal losses is $e_{\text {start }}=L_{f} \times P_{g, d} \times t$

vi) The plant will start generating electricity only after the $e_{\text {start }}$ compensation is completed.

vii) Corresponding to $f_{t h}$, the fraction of rated gross power $f_{p}$ is computed (See Fig. 4 \& Fig. 5).

\footnotetext{
${ }^{1}$ Maximum value of $f_{h b}$, the fraction of thermal power that can be used is limited in our analysis to 0.2.
} 
viii) Corresponding to $f_{p}$ the maximum gross electrical energy $e_{g}$ (in the absence of thermal losses) that could be generated is computed. However the actual electrical energy generated $\left(e_{g, a}=e_{g}-\right.$ $\left.e_{s t a r t}\right)$. Then the corresponding fractional power generated $\left(f_{p a}\right)$ is $e_{g, a} / P_{g, d}$, as $e_{g, a}$ is for one hour.

ix) Then considering the auxiliary power requirements, the electrical energy supplied to grid $\left(e_{\text {grid }}\right)$ during that hour can be calculated. Auxiliary power fraction is the ratio of power consumed by the auxiliary units to the gross power generated (default value is 0.1 ).

$\mathrm{x}) \quad$ The sum of the net electrical energy generated ( $\left.\sum e_{\text {grid }}\right)$ over 8760 hours gives the annual electrical energy generated by the plant.

xi) The energy share of hybridization at each hour is estimated as:

$$
e_{h b}=\frac{f_{h b, u s e d}}{f_{\text {th }}} \times e_{\text {grid }}
$$

where, $f_{h b, u s e d}$ is the fraction of thermal power utilized from hybridization

xii) The annual energy attributed to the solar is $E_{s}=\sum e_{s}=\sum e_{\text {grid }}-\sum e_{h b}$

xiii) The capacity factor of the plant is estimated as below

$$
\text { Capacity Factor }=\sum e_{g, a} / P_{g, d} \times 8760
$$

xiv) The annual efficiency $\left(\eta_{s-e}\right)$ of conversion from solar to electric energy is as follows.

$$
\eta_{s-e}=\sum e_{s} /\left\{A_{r} \times S M \times \sum(D N I \times \cos \theta)\right\}
$$

\section{Validation of the model}

Based on the above methodology, we have developed a java based Graphic User interface (GUI) tool, CSTEM CSTEP's Solar Techno-Economic Model (For details visit: www.cstep.in ). It is to facilitate hourly simulation of CSP plants with trough technology for various capacities considering thermal storage and hybridization.

Table 1 shows the validation of our model with the real data from two parabolic trough solar plants viz., Nevada Solar Electric Generating Station VI (SEGS-VI) (NREL, 2013 $)$ and Solana Generating Station (NREL, 2013 $3^{\mathrm{b}}$ ). The CSTEM's results for SEGS-VI plant are corresponding to SM=1.3184 and for Solana Generating Station are corresponds to $\mathrm{SM}=1.9332$. The SM in the CSTEM model is chosen such that the solar field area used in the model is the same as that used in the plants. It is obvious that SEGS-VI has a smaller SM as it has no thermal storage but Solana plant has higher SM as it incorporates six hours thermal storage. The DNI data used for the analysis is a typical TMY file (a representative year for 15 to 20 years) available for that sites corresponding to the two plants. Since TMY is used for the analysis, the average electrical energy is considered for the comparison. The electrical energy values estimated also depends on the part load performance of the turbine. Therefore, it can be observed from Table 1 that the electrical energy generated by the two plants is agreeable though with the error of $11 \%$ with the CSTEM's results.

\section{Results and Discussion}

The results of the engineering model described in Section 2.3, applied to some typical cases are discussed in this section. Jodhpur, India is considered as a location for case study. The latitude of the location is 26.26 degrees. The hourly DNI data developed by CSTEP is used in this analysis (Ramaswamy et al, 2013). The parameters chosen for the study are given in Table 2. 
The annual electrical energy generated, capacity factor, annual efficiency etc. have been computed for different combinations of the above parameters for a range of SM from one to four.

\subsection{Case-1: No thermal storage and No Hybridization}

\subsubsection{Effect of Capacity of the Plant}

Table 3 gives the variation of power block efficiency under design conditions, reference mirror aperture Area and reference mirror aperture area per MW of the capacity of the plant.

It can be seen that the design efficiency of the power block increases with capacity (see Fig.3) and consequently the reference aperture area/MW decreases with increase in capacity. This trend is illustrated in Fig 6. From this, it is obvious that lower capacity plants will be expensive.

\subsubsection{Effect of Solar Multiple}

The variation of the annual electrical energy generated can be expressed in terms of annual energy per MW capacity since the annual electrical energy generation is directly proportional to the plant capacity. Fig.7 shows this variation with SM. The variation of capacity factor with SM is shown in Fig.8. From these figures, it is clear that increasing SM beyond three is only marginally beneficial.

The annual efficiency vs. SM is plotted in Fig.9 for various capacities of the plant. From this figure, one can infer that the maximum efficiency occurs around $\mathrm{SM}=1.5$ for all capacities and this annual efficiency increases with capacity.

\subsubsection{Electrical Power Generation during a Typical Day}

Fig. 10 gives the variation of $f_{t h s}$ and $f_{p}$ during a typical day ( $21^{\text {st }}$ January), for $\mathrm{SM}=1.0$ and $\mathrm{SM}=2.0$. The advantage of representing the data in terms of $f_{t h, s}$ and $f_{p}$ is that, it is independent of the capacity of the plant. One can notice the lag in the generation of electric power relative to the solar power, to account for making up of the thermal losses that would have occurred overnight. The solar power over the initial period is used up to make up the losses and bring the HTF to operating conditions, before the power generation can occur.

For $\mathrm{SM}=1.0, f_{p}$ is slightly less than $f_{t h, s}$, due to lower efficiency of the power block during part load conditions $\left(f_{p}=1.0\right.$, when $\left.f_{t h, s}=1.0\right)$. For $\mathrm{SM}=2.0, f_{t h, s}$ is twice the value corresponding to $\mathrm{SM}=1.0$. However, the $f_{p}$ gets limited to 1.1 , as the plant is permitted to work with $10 \%$ overload. So the excess solar power cannot be utilized and the mirrors have to be suitably defocused to $\operatorname{limit} f_{p}=1.1$.

\subsection{Case-2: Thermal Storage without Hybridization}

\subsubsection{Effect of Solar Multiple}

The variation of annual electrical energy generated with SM, with three hours and six hours of thermal storage for various capacities of the plant were computed and therefore annual electrical energy generated per MW capacity is plotted versus SM in Fig. 11. It was found that the annual electrical energy generated is proportional to rated capacity even with storage. Hence, the trend is similar even for capacity factor with SM for various storage hours. Capacity factor is also presented in terms of its variation with thermal storage for various SM in Fig. 12. One can infer from these figures that providing thermal storage without sufficient SM is not beneficial. Higher requirement of thermal storage hours implies the need for higher SM.

The annual solar to electric efficiency for six hours of thermal storage with SM, for various capacities, is shown in Fig.13. It can be seen from this figure that for three hours of thermal storage, the highest efficiency occurs around $\mathrm{SM}=2.2$. This is the optimum value of SM from an energy efficiency point of view. However, from economical point of view, the optimum SM has to be determined based on least $L C O E$.

\subsubsection{Electrical Power Generation during a Typical Day}

Fig. 14 shows the variation of $f_{t h, s}$ and $f_{p}$ during a typical day for $\mathrm{SM}=2 \& 2.2$ and for six hours of thermal storage. For $\mathrm{SM}=2$ and $t_{s}=6$, the electrical power generated does not go for six hours after the sun sets, but slightly close to three hours and indicating that $\mathrm{SM}=2.0$ is not sufficient to cater for six hours of thermal storage. With $\mathrm{SM}=2.2$, the thermal storage capacity is better utilized and power is generated for about four 
hours. It must however be stated that such inferences should not be based on the performance over a single day, but must be based on the annual efficiency as indicated earlier. The data on the variation of $f_{t h, s}$ and $f_{p}$ during a day have been shown to give some physical idea of how thermal storage, with appropriate SM, helps in extending the power plant operation beyond sunshine hours.

\subsection{Case-3: Hybridization without Thermal Storage}

\subsubsection{Effect of Solar Multiple}

The hybridization used in this analysis is to augment the thermal power to HTF to the extent of 0.1 and 0.2 times the design thermal power, so that the electrical energy produced is as high as possible. Fig. 15 shows for $\mathrm{SM}=1.0$, the variation in $f_{p a}$ during a day for $f_{h b}$ is $0,0.1$ and 0.2 along with $f_{t h, s}$. Fig. 16 gives the value of $f_{h b, u s e d}$ during the day. It may be noticed that $f_{p a}$, the fractional gross power generated increases with $f_{h b}$. $f_{h b, u s e d}$ is equal to $f_{h b}$ through-out the day. The benefit of hybridization for $\mathrm{SM}=1$ is maximum.

Fig. 17 shows for $\mathrm{SM}=1.5$, the variation in $f_{p}$ during a typical day for $f_{h b}=0,0.1$ and 0.2 along with $f_{t h, s}$. Fig. 18 gives the value of $f_{h b \text {,used }}$ during the day. It may be noticed that the power generated $f_{p}$ is nearly the same for all the three cases except during the first hour in the morning and the last hour in the evening. With SM=1.5, the solar power $f_{\text {th,s }}$ available during most of the day is more than that required to generate even $10 \%$ more than rated electrical power. Therefore hybridization is not required for most of the time. Thus hybridization is utilized during the first hour in the morning and during the last hour in the evening.

It is observed from Fig. 17 that in the first hour $f_{t h, s}$ and hybridization contribute towards making up the thermal losses that would have occurred overnight. So $f_{p a}$ is very small during the first hour in the morning, whereas it is much higher in the last hour.

Since hybridization does not contribute much for even $\mathrm{SM}=1.5$, higher values of $\mathrm{SM}$ were not considered.

\subsubsection{Effect of Hybridization on Annual Efficiency Attributed to Solar Field}

Hybridization not only increases the electrical energy generated due to additional thermal energy input but also increases the electrical energy attributable to the solar field because of higher efficiency at higher part load operation. The variation of annual efficiency with SM for $f_{h b}=0,0.1$ and 0.2 is shown in Fig. 19 for 1 MW and $50 \mathrm{MW}$ capacities. From this figure, it is clear that the annual efficiency increases with $f_{h b}$ but the benefit of hybridization decreases as SM increases.

\subsection{Case-4: With Thermal Storage and Hybridization}

It was mentioned earlier that the capacity factor is independent of the rated capacity of the plant. Fig.20 and Fig. 21 show respectively the variation of capacity factor with SM for zero and six hours of thermal storage. In each figure, hybridization values of $0,0.1$ and 0.2 are considered. It is seen that capacity factor increases with $\mathrm{SM}$ but the benefit of hybridization is maximum for $\mathrm{SM}=1$ and decreases as $\mathrm{SM}$ increases.

\section{Summary}

The optimum SM's with respect to the annual efficiency for different plant capacities and hours of thermal energy storage for no hybridization case is summarized and given in Table 4. The role of hybridization is significant only when there is no thermal energy storage and lower SM's. The optimum SM for maximum hybridization considered is around 1.2-1.3 for any plant capacity.

\section{Conclusion}

One of the needs for a detailed step by step approach to design a solar plant using trough technology using the concept of Solar Multiple has been discussed in this paper. Also, the use of thermal energy storage and hybridization to enhance the utility of a solar plant has been deliberated. This also provides a method to arrive at the reference aperture area of the solar field for given plant capacity and location. The tool (CSTEM) developed based on this methodology facilitates to determine the optimum solar multiple for which the electrical energy 
generated is maximum. In the absence of good data on cost estimates, this is a good indication of optimized solar field. We have found that, for Jodhpur, the optimum solar multiples for a plant with zero hours, three hours and six hours thermal energy storage and no hybridization are $1.4,1.8$ and 2.4 respectively which is independent of plant capacity. The annual efficiency of a plant increases for higher capacity plants. The efficiencies are approximately about $10 \%$ for a $1 \mathrm{MW}$ plant and $20 \%$ for a $50 \mathrm{MW}$ plant without storage and hybridization. Having six hours of thermal energy storage the plant efficiency increases by $2 \%$. It was observed that hybridization is effective at lower Solar Multiples. Hybridization improves the efficiency of plant by operating the power block at better part load conditions.

It is recommended that the CSTEM tool can be used by designers, developers and policy makers for carrying out the preliminary design to estimate the optimum size of the solar field for a parabolic trough based solar field with thermal storage and hybridization for plant capacities ranges from $1 \mathrm{MW}$ to $50 \mathrm{MW}$ at any location.

\section{Acknowledgements}

The base version to develop the CSTEM tool work was supported by Ministry of New \& Renewable Energy, Government of India (Vide letter No. 15/26/2010-11/ST). Later the tool was upgraded with the support of the US-India Partnership to Advance Clean Energy-Research (PACE-R) for the Solar Energy Research Institute for India and the United States (SERIIUS), funded jointly by the U.S. Department of Energy and the Government of India, through the Department of Science and Technology under Subcontract IUSSTF/JCERDC-SERIIUS/2012. The authors are grateful to Anshu Bharadwaj for his constant encouragement, support and guidance. The authors are also thankful to $\mathrm{N}$ Balasubramanian and $\mathrm{N}$ Ramakrishnan for their critical review in improving the manuscript. 


\section{References}

Adbelkader Zaaraoui, Mohamed Lamine Yousfi, Noureddine Said. 2012. Technical and economical performance of parabolic trough collector power plant under algerial climate. Procedia Engineering. 33, 78-91.

N.S. Suresh, Badri. S. Rao, Srilakshmi G, Thirumalai N C, M.A. Ramaswamy. 2014. Steady state heat transfer analysis for a parabolic trough collector. Center for Study of Science Technology and Policy (CSTEP).

Feldhoff JF, Schmitz K, Eck M, Schnatbaum-Laumann L, Laing D, Ortiz-Vives F, Schulte-Fischedick J. 2012. Comparative system analysis of direct steam generation and synthetic oil parabolic trough power plants with integrated thermal storage. Solar Energy. 86, 520-530

Fichtner. 2010. Assessment of Technology Options for Development of Concentrating Power in South Africa. The world Bank \& ESMAP. Available at <www.climateinvestmentfunds.org/cif/sites/climateinvestmentfunds.org/files/Presentation\%20\%20WB\%20(Eskom)\%20Project\%20-\%202010_12_07\%20.pdf> accessed on April,2013.

Forristall. 2003. Heat trasnfer analysis and modeling of a parabolic trough solar reciever implemented in engineering equation solver. Report No. NREL/TP-550-34169, NREL.

Franchini G, Perdichizzi A, Ravelli S, Barigozzi G. 2013. A A comparative study between parabolic trough and solar tower technologies in Solar Rankine Cycle and Integrated Solar Combined Cycle plants. J. Sol. Energy. 98, 302-314.

Gharbhi Nanla El, Halima Derbal, Sofiane Bouaichaoui, Noureddine Said. 2011. A comparative study between parabolic trough collecot and linear fresnel reflector technologies. Energy Procedia. 6, 565-572.

Garcia IL, Alvarez JL,Blance D. Performance model for parabolic trough solar thermal power plants with thermal storage: Comparision to operating plant data. 2011. Solar Energy. 85, 2443-2460.

Gee R, Murphy LM. Near-term improvements in parabolic troughs:an economic and performance assessment. 1981. Solar Energy Research Institute, Colorado.

Geostri Andrea, Macro Binotti, Macro Astolfi, Paolo Silva, Ennino Machhi, Giapaolo Manzolini. 2012. Comparision of different solar plants based on paraboilic trough technology. J. Sol. Energy. 86, 1208-1221.

Hachicha A A, Rodriguez I, Capdevila R, Olive A. 2013. Heat transfer analysis and numerical simulation of a parabolic trough solar collector. Applied Energy. 111,581-592.

Herrmann Ulf, Bruce Kelly, Henry Price. Two-tank molten salt storage for parabolic trough solar power plants. 2004. J.Energy. 29, 883-893.

Huang W, Hu P, Chen Z. 2012. Performance simulation of a parabolic trough solar collector. Sol. Energy. 86, 746-755.

IRENA. 2012. Renewable energy technologies: Cost analysis series. Vol.1: power Sector, Issues 2/5. International Renewable Energy Agency (IRENA).

Keith Lovegrove, Muriel Watt, Robert Passey, Grame Pollock, Joe Wyder, Josh Dowse. Realising the potential for concentrating solar power in Australia. 2012. IT Power (Australia) Pvt Ltd. 
Kuravi Sarada, Jamie Tranhan, D Yogi Goswami, Muhammad M Rahman, Elias K Stefanakos. 2013. Thermal energy storage technoliges and systems for concentrating solar power plants. 39, 285-319.

Lippke,F. 1995. Simulation of the part-load performance of a 30 Mwe SEGS Plant. Report No. SAND951293. Sandia National Labs. Alburquerque, USA.

Montes MJ, Abanades A, Martinez Val, Valdes M. 2009. Solar multiple optimization for a solar-only thermal power plant, using oil as heat trasnfer fluid in the parabolic trough collectors.Solar. Energy. 83, 2165-2176.

Montes MJ, Rovira A, Munoz M, Martinez-Val JM. 2011. Performance analysis of an Integrated Solar Combined Cycle using Direct Steam Generation in parabolic trough collectors. J. Applied Energy. 88. 32283238.

NREL. 2013 ${ }^{\mathrm{a}}$. Concentrating Solar Power Projects: Solar Electric Generating Station. Available at <http://www.nrel.gov/csp/solarpaces/project_detail.cfm/projectID=33> accessed on May, 2013.

NREL. 2013 ${ }^{\mathrm{b}}$. Concentrating Solar Power Projects: Solana Generating Station. Available at < http://www.nrel.gov/csp/solarpaces/project_detail.cfm/projectID=23> accessed on May, 2013.

Patnode, A.M. 2006. Simulation and performance evaluation of parabolic trough solar power plants. Ph.D Thesis. University of Wisconsis, USA.

Pilkington. 1996. Status report on solar trough power plants - Experience, prospects and recommendations to overcome market barriers of parabolic trough collector power plant technology. Pilkington Solar International $\mathrm{GmbH}$, Germany.

Powell M Kody, Edgar F Thomas. 2012. Modeling and control of a solar thermal power plant with thermal energy storage. Chem. Engg., Science. 71, 138-145.

Ramaswamy MA, Badri S Rao, Thirumalai NC, Suresh N S. Estimation of hourly direct normal irradiance for 22 stations in India revised. 2013. Center for Study of Science Technology and Policy (CSTEP).

Ravaghi-Ardebilli Zohreh, Flavio Maneti, Nadson M N Lima, Lamia Zuniga Linan. 2013. Study of direct thermal energy storage technologies for effectiveness of concentrating solar power plants. The Italian Association of Chemical Engineering (AIDIC). 32.

Ravi Kumar K, Reddy K S. 2009. Thermal analysis of solar parabolic trough with porous disc receiver. Applied Energy. 86, 1804-1812.

Reddy K S, Ravi Kumar K. 2012. Solar collector field design and viability analysis of stand-alone parabolic trough power plants for Indian conditions. Energy for sustainable development. 16 (4), 456-470.

Schiricke B, Pitz-Paal R, Lupfert E, Pottler K, Pfander M, Riffelmann KJ, Newmann A. 2009. Experimental verification of optical modeling of parabolic trough collectors by flux measurement. J.Sol.Energy Eng., 131,011004:1-6.

Thomas A, Guven H M. 1993. Parabolic trough concentrators - design, construction and evaluation. Energy Convers. Mgnt. 34, 401-416. 


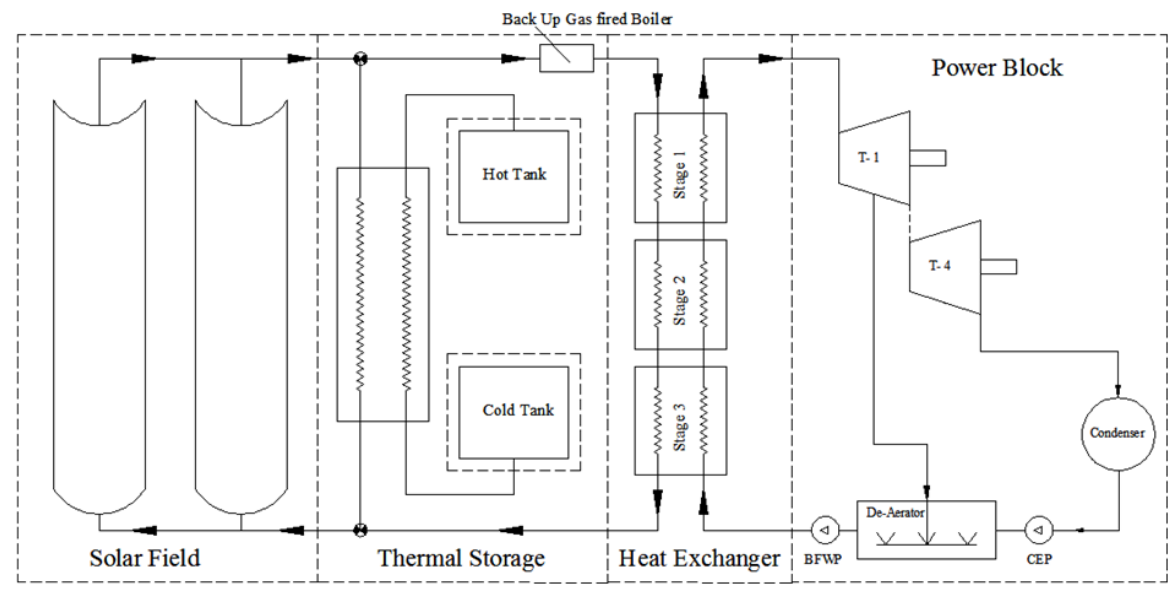

Fig. 1. Schematic representation of the parabolic trough plant with thermal storage and hybridization

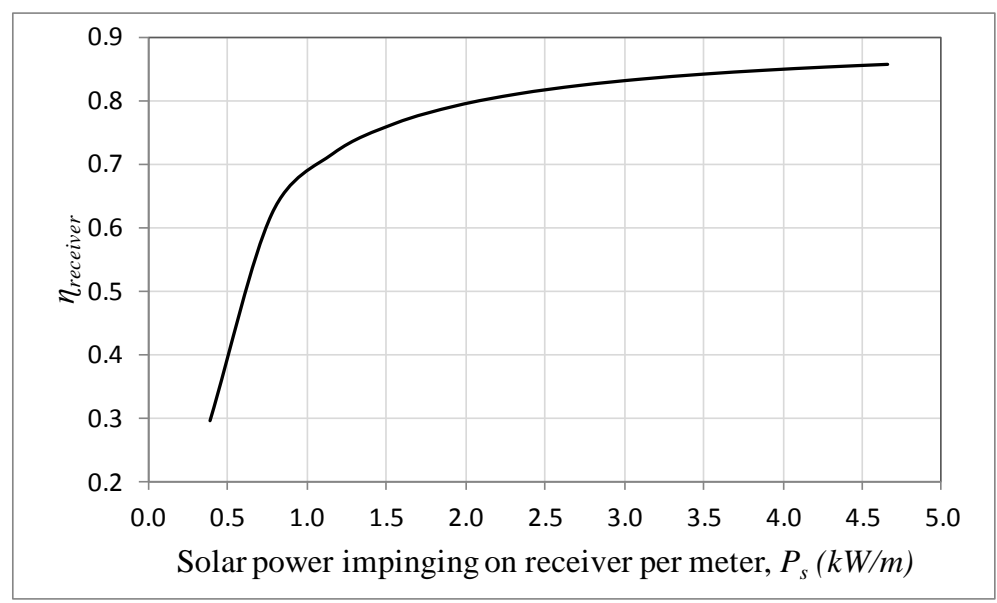

Fig.2: Receiver efficiency as a function of input solar power that is reflected from the trough aperture

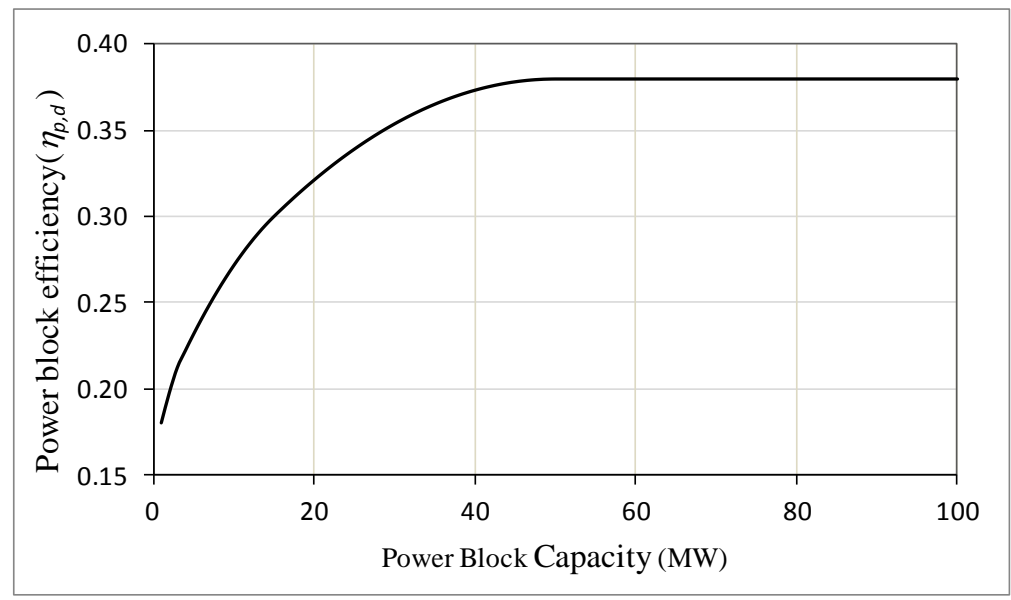

Fig.3: Power block efficiency at design conditions 


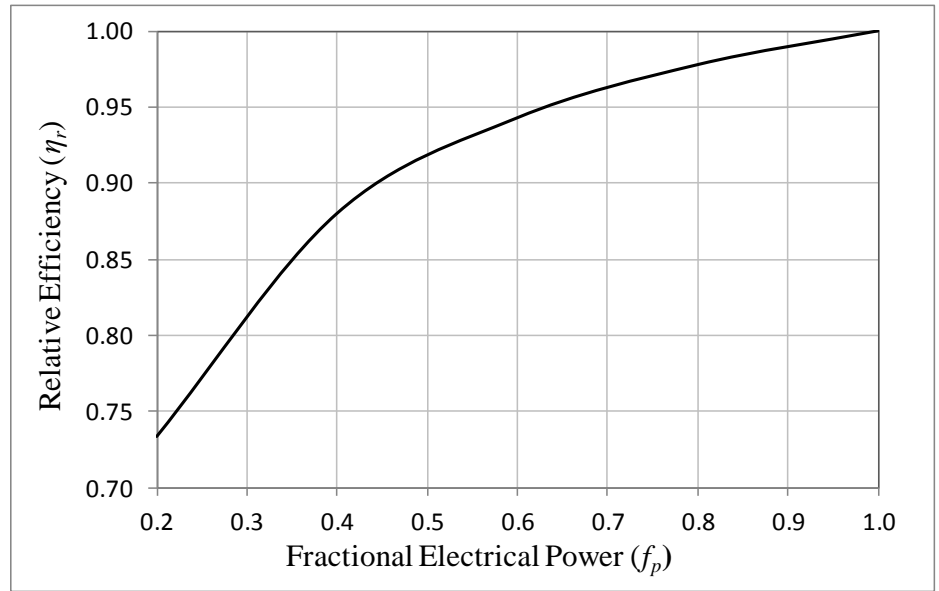

Fig. 4: Efficiency of power block at part load

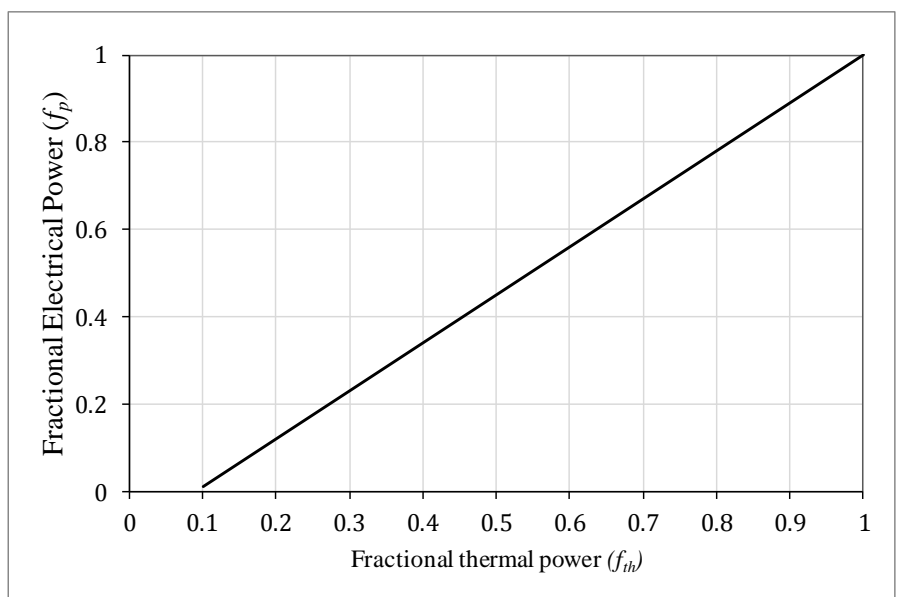

Fig.5: Electrical power generated at part load conditions

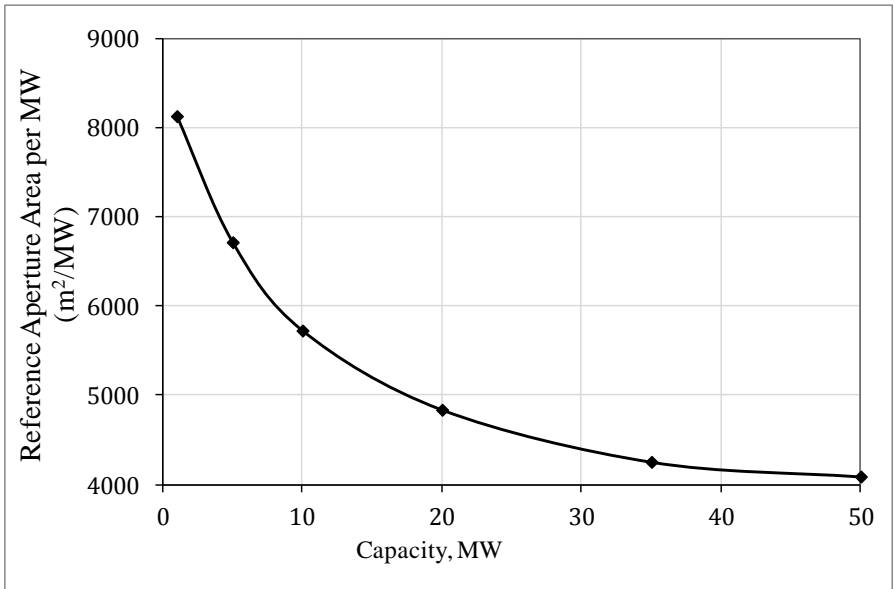

Fig 6: Variation of reference mirror aperture area per MW with capacity 


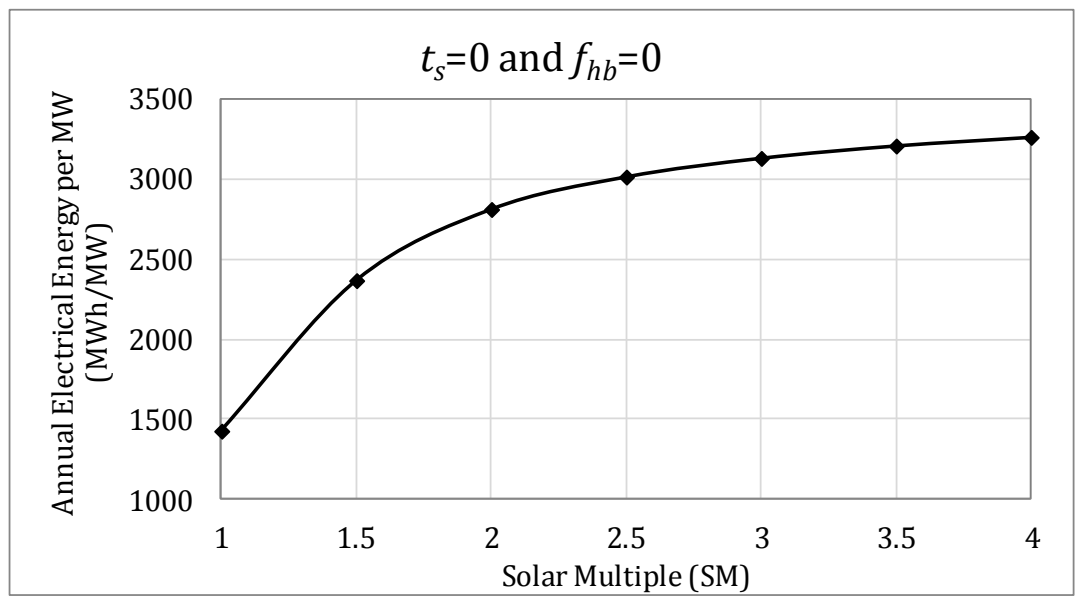

Fig.7: Variation of annual electrical energy generated per MW with SM

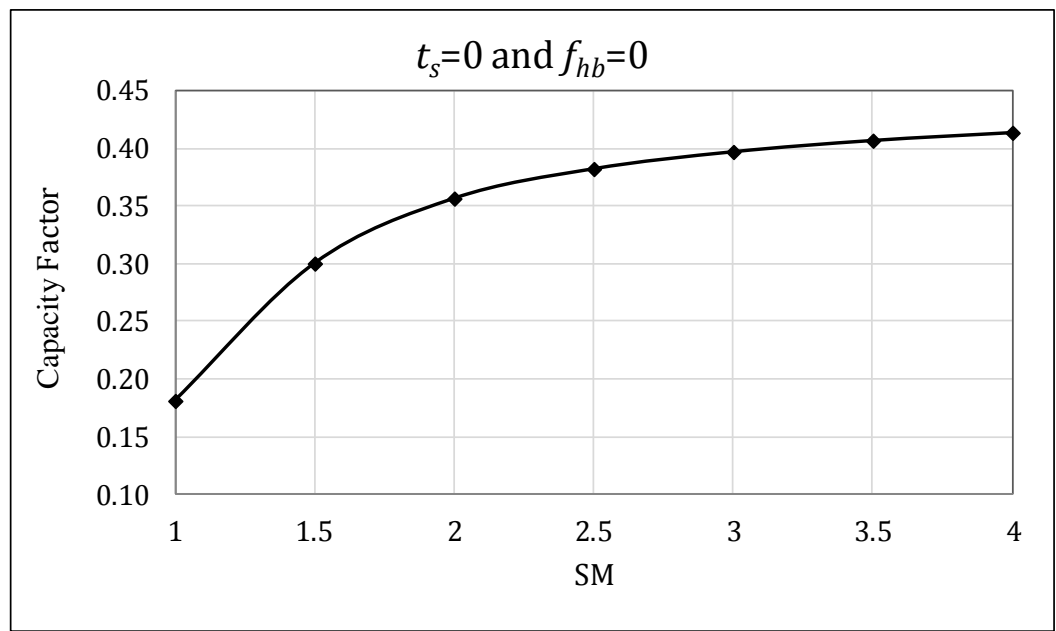

Fig.8: Variation of annual electrical energy generated per MW with SM

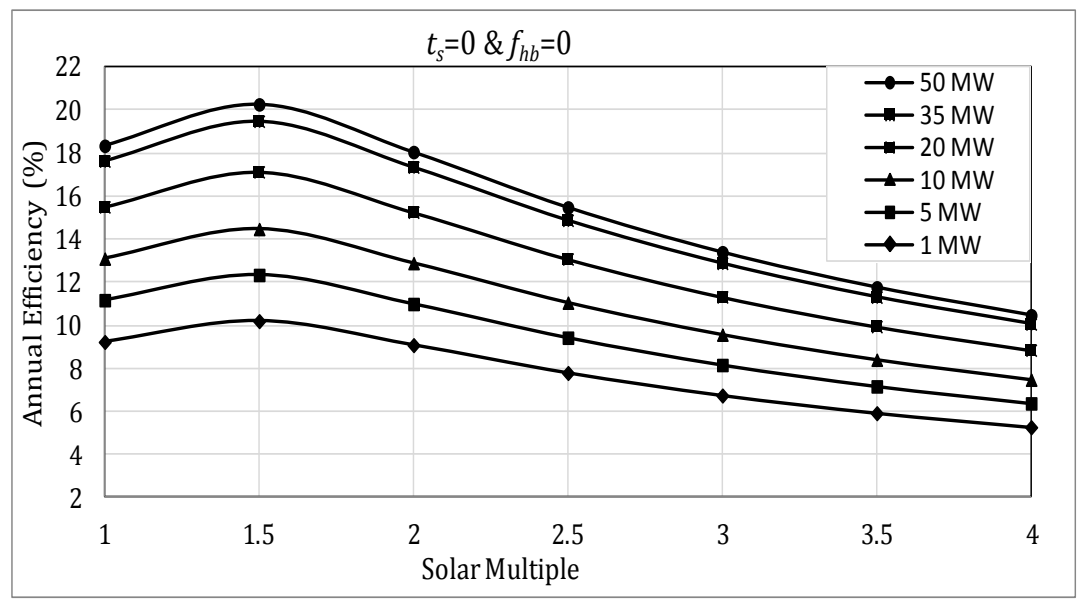

Fig.9: Variation of overall annual efficiency with SM for various capacities 


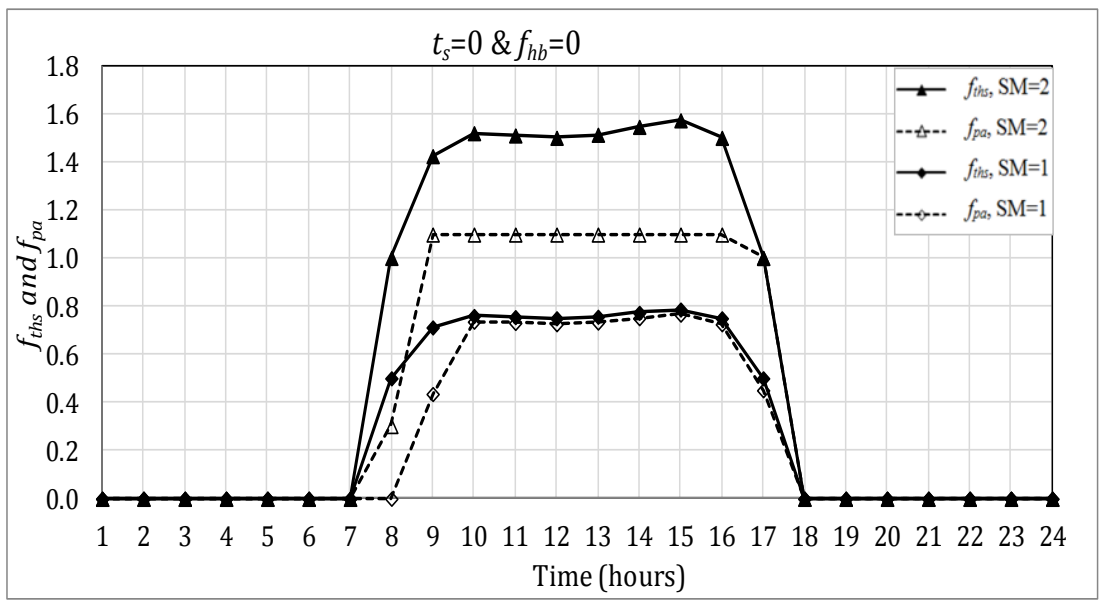

Fig.10: Variation of the fractional solar thermal power and fractional electrical power generated during a typical day

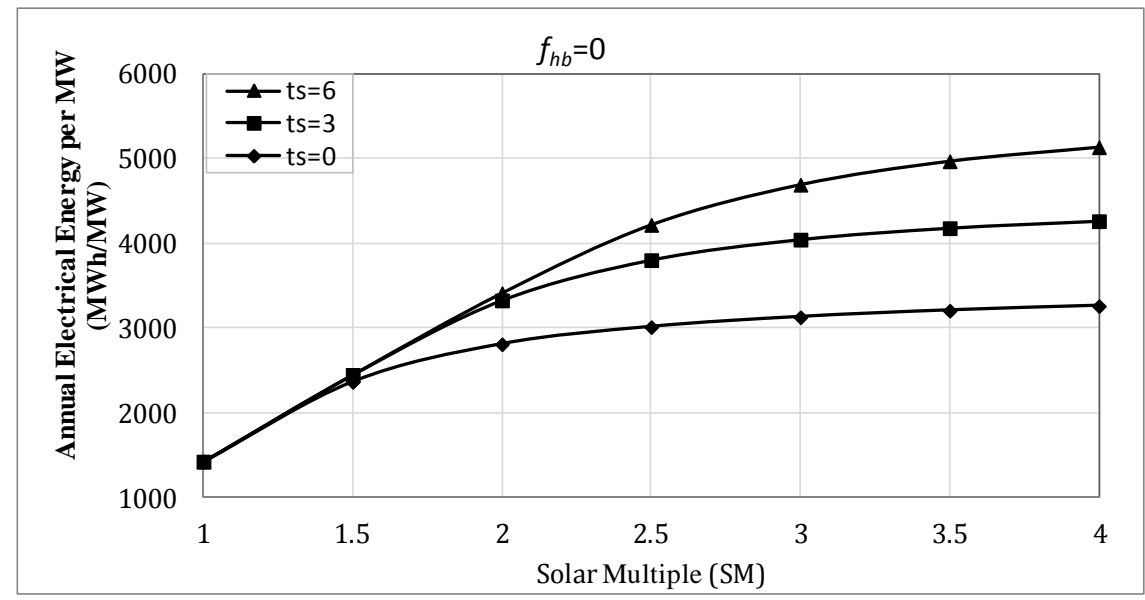

Fig. 11: Variation of annual electrical energy/MW with SM for $t_{s}=0,3$ and 6 hours

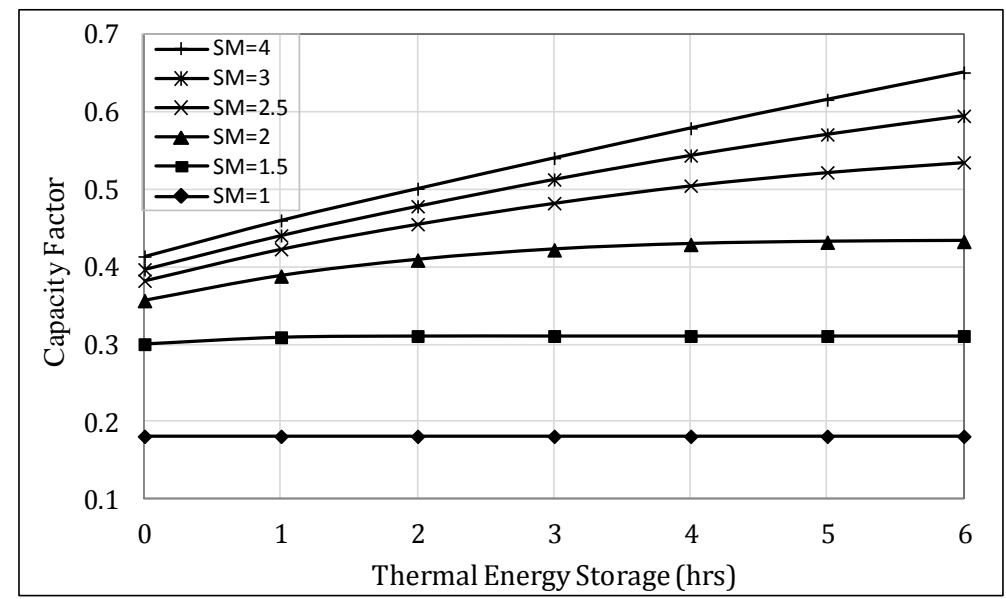

Fig. 12: Variation of capacity factor with thermal energy storage for various SMs and no hybridization 


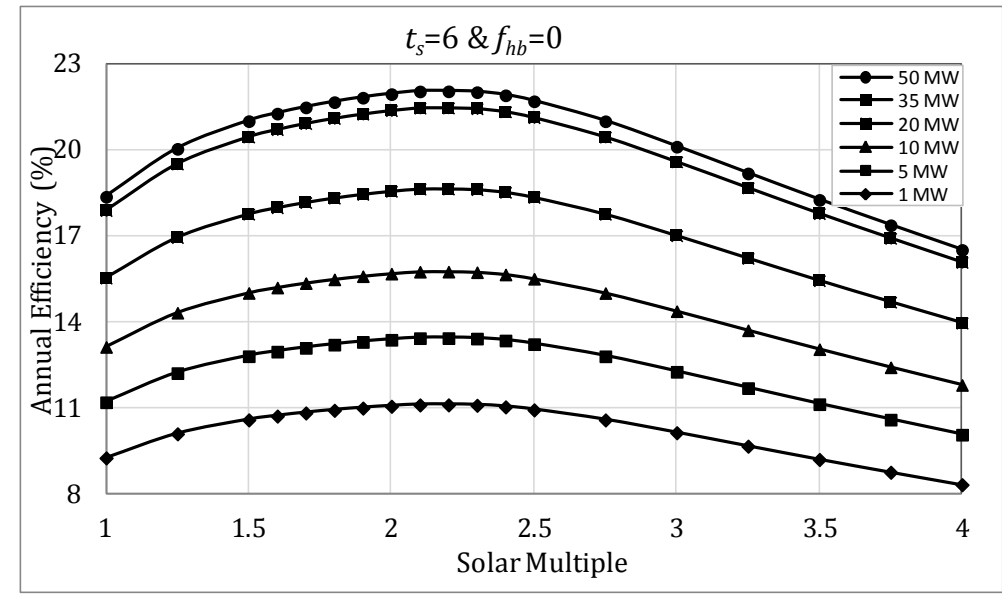

Fig.13: Variation of overall annual efficiency with SM for various capacities for $t_{s}=6$ hours and no hybridization

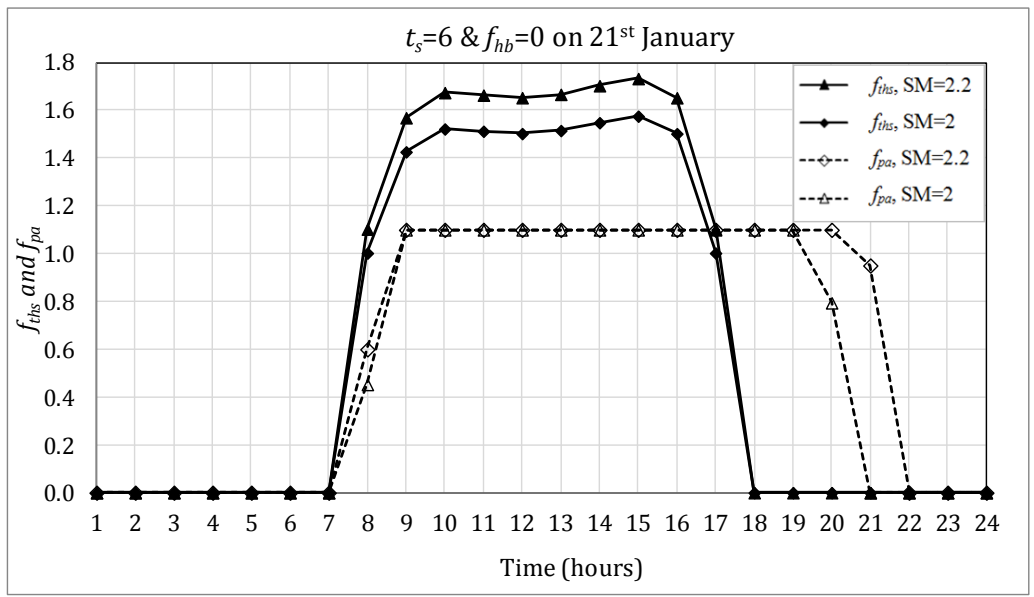

Fig. 14: Variations in $f_{t h, s}$ and $f_{p a}$ during a typical day for $t_{s}=6$ hours

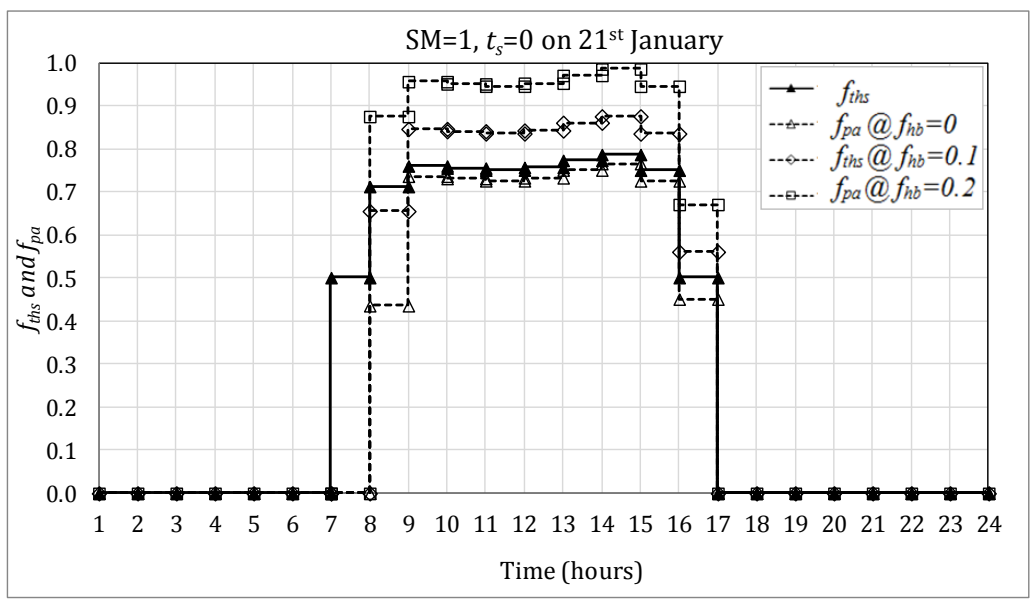

Fig.15: Effect of hybridization on the electrical power generated during a typical day for $\mathrm{SM}=1$ 


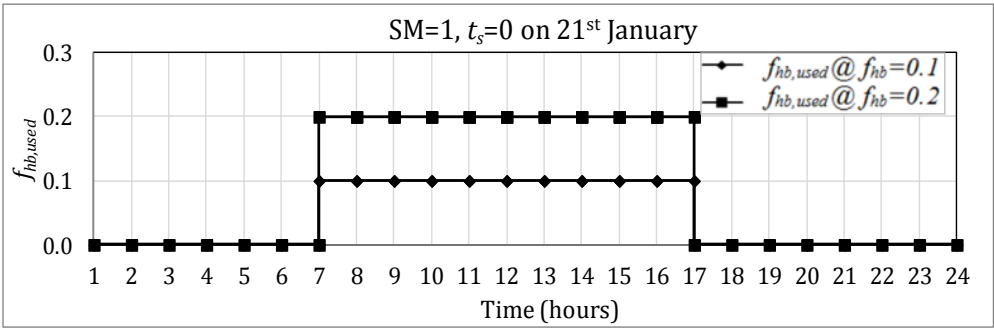

Fig.16: Fraction of hybridization used during a typical day for $\mathrm{SM}=1$

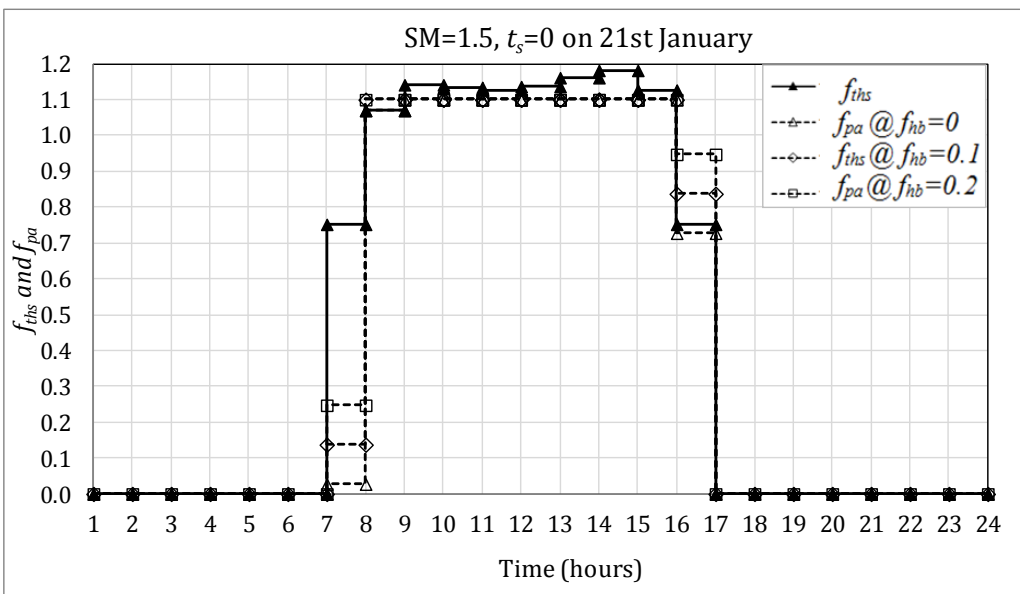

Fig.17: Effect of hybridization on the electrical power generated during a typical day for SM=1.5

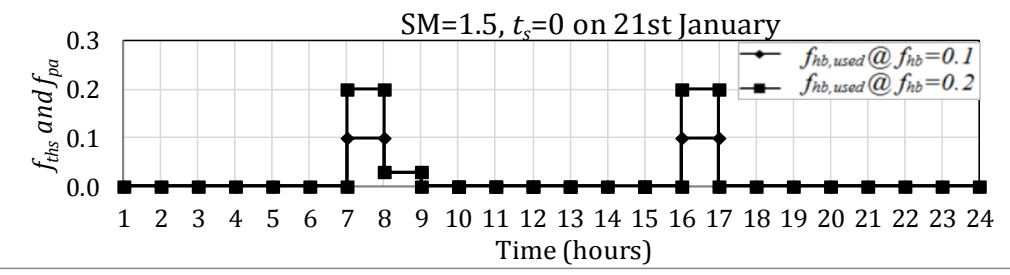

Fig. 18: Fraction of hybridization used during a typical day for $S M=1.5$

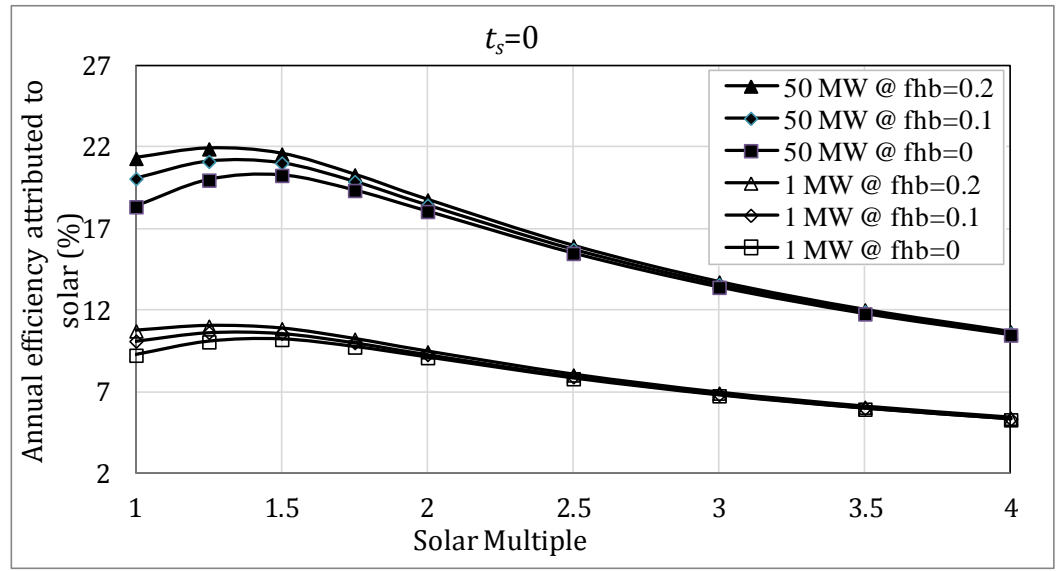

Fig.19: Variation of annual efficiency attributed to the solar with SM for $f_{h b}=0,0.1$ and 0.2 


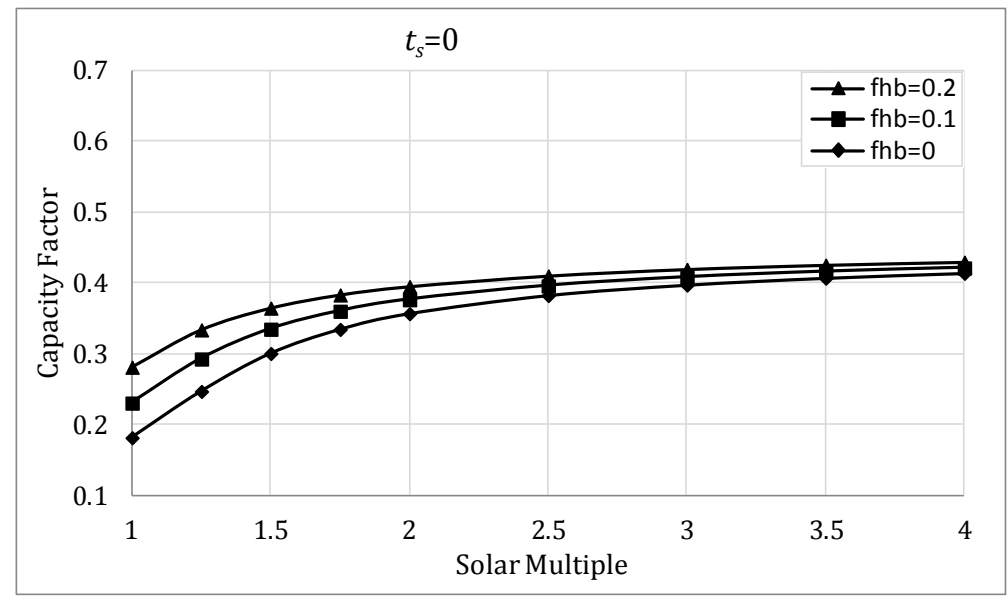

Fig. 20: Variation of capacity factor with $S M$ for $t_{s}=0$ hours

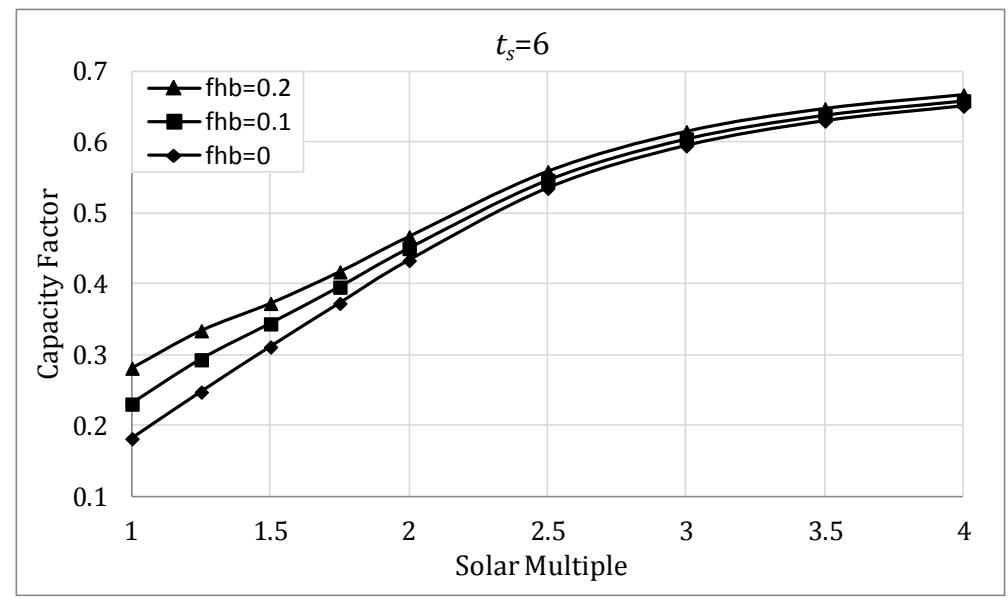

Fig.21: Variation of capacity factor with SM for $t_{s}=6$ hours 
Table 1: Validation of CSTEM with plants data

\begin{tabular}{|c|c|c|c|c|c|c|}
\hline \multirow[t]{2}{*}{ Parameters } & \multicolumn{3}{|c|}{$\begin{array}{c}\text { SEGS-VI (35 MW, } 0 \text { hours } \\
\text { storage, } 5 \text { MW Auxiliary \& } \\
\text { 1.9E5 } \mathrm{m}^{2} \text { of solar field) }\end{array}$} & \multicolumn{3}{|c|}{$\begin{array}{c}\text { Solana Generating Station } \\
\text { (280 MW, } 6 \text { hours storage, } 30 \\
\text { MW Auxiliary \& } 2.2 \mathrm{E} 6 \mathrm{~m}^{2} \text { of } \\
\text { solar field) }\end{array}$} \\
\hline & $\begin{array}{l}\text { Plant } \\
\text { Data }\end{array}$ & $\begin{array}{l}\text { CSTEM } \\
\text { Results }\end{array}$ & Error & $\begin{array}{c}\text { Plant } \\
\text { data }\end{array}$ & $\begin{array}{l}\text { CSTEM } \\
\text { Results }\end{array}$ & Error \\
\hline Electrical energy generated, MWh & $67758^{*}$ & 76533 & $+11.46 \%$ & $0.944 \mathrm{E}^{+}$ & $1.069 \mathrm{E} 6$ & $+11.65 \%$ \\
\hline
\end{tabular}

Table 2: Parameters considered for performance assessment

\begin{tabular}{ll}
\hline Gross capacity (MWe) & $1,5,10,20,35,50$ \\
Thermal energy storage (hours) & $0,3,6$ \\
Percentage of hybridization (Fraction of design thermal power) & $10,20,30$ \\
\hline
\end{tabular}

Table 3: Variation in power block efficiency \& reference aperture area with capacity

\begin{tabular}{cccc}
\hline Capacity $(M W)$ & $\boldsymbol{\eta}_{\boldsymbol{p}, \boldsymbol{d}}$ & $\boldsymbol{A}_{\boldsymbol{r}}\left(\mathrm{m}^{2}\right)$ & $\boldsymbol{A}_{\boldsymbol{r}} \boldsymbol{p e r} \mathbf{M W}\left(\mathrm{m}^{2} / M W\right)$ \\
\hline 1 & 0.19 & 8132 & 8132 \\
5 & 0.23 & 33590 & 6718 \\
10 & 0.27 & 57281 & 5728 \\
20 & 0.32 & 96804 & 4840 \\
35 & 0.37 & 148963 & 4256 \\
50 & 0.38 & 204575 & 4091 \\
\hline
\end{tabular}

Table 4: Variation of maximum overall annual efficiency and corresponding optimised SM with plant capacity for ts $=0,3$ and 6

\begin{tabular}{|c|c|c|c|c|c|c|}
\hline \multirow{2}{*}{$\begin{array}{c}\text { Capacity } \\
\text { (MW) }\end{array}$} & \multicolumn{2}{|r|}{$t_{s}=0$} & \multicolumn{2}{|r|}{$t_{s}=3$} & \multicolumn{2}{|r|}{$t_{s}=6$} \\
\hline & SM & $\eta_{\text {annual }}(\%)$ & SM & $\eta_{\text {annual }}(\%)$ & SM & $\eta_{\text {annual }}(\%)$ \\
\hline 1 & \multirow{6}{*}{1.4} & 10.2 & \multirow{6}{*}{1.8} & \begin{tabular}{|l|}
10.71 \\
\end{tabular} & \multirow{6}{*}{2.3} & 11.07 \\
\hline 5 & & 12.34 & & 12.97 & & 13.39 \\
\hline 10 & & 14.48 & & 15.21 & & 15.71 \\
\hline 20 & & 17.13 & & 18.00 & & 18.59 \\
\hline 35 & & 19.48 & & 20.47 & & 21.41 \\
\hline 50 & & 20.27 & & 21.3 & & 21.99 \\
\hline
\end{tabular}

